\title{
Electro-Mechanical Challenges in the Design of a High-Speed-High-Power-PMSM Rotor for an Aerospace Application
}

\author{
Nicola Chiodetto, Barrie Mecrow, Rafal Wrobel and Timothy Lisle \\ Newcastle University, School of Engineering, Newcastle upon Tyne (UK)
}

\begin{abstract}
This paper deals with the electro-mechanical design of a high-speed-high-power (HSHP) electric motor. The focus is twofold: both mechanical and electromagnetic aspects are of interest. The former regards holding forces in order to sustain the retaining sleeve, components stresses, and rotordynamic behaviour of the rotor assembly. The latter addresses eddy current losses arising in different rotor parts. Usually different physics involving rotor losses, mechanical and thermal stresses are treated separately or superficially analysed with rules of thumbs approaches.This work addresses the lack of a comprehensive more detailed design treating mechanics and electromagnetic challenges together. The rotor under study is for an aerospace propulsion application known as Boundary-LayerIngestion (BLI).

Index Terms-BLI, rim-driven, high speed-high power surface permanent magnet, multi-physics, eddy-currents, rotor-dynamics
\end{abstract}

\section{INTRODUCTION}

Aircraft hybrid-electric-propulsion solutions are being actively investigated. One of the most interesting is represented by Boundary Layer Ingestion (BLI): it provides an augmentation of thrust and a drag reduction, decreasing the overall aircraft fuel consumption. Air streams along the fuselage (boundary layers) are accelerated by additional fans, mounted near the aircraft body rear providing maximum drag reduction as the air streams are more intense in this zone. Usage of electric motors for driving a multiple fan system becomes advantageous if the drive-motor is high power dense. The easiest way to achieve this is increasing rotational speed and electric frequency [1]. However, a speed/frequency increment does not come without side effects. Mechanical issues arise, regarding rotor strength capabilities and rotor-dynamic instabilities. High Speed operation introduces considerable mechanical stress due to centrifugal forces. Simultaneously, parasitic electromagnetic losses arise in rotating conductive components. Therefore, the modelling of any high-speed-highpower (HSHP) electric motor is multi-physics, by nature. Less conventional solutions to limit losses are employed in this type of motor, like electromagnetic screening. Literature on shielding of permanent magnet motors is limited, although some studies have been undertaken in the past [2], [3] and more recently [4], [5]. These studies perform an approximate analysis of the high order effects encountered in the shielding behaviour. Most of them do not consider possible implications affecting rotor mechanics as the design is made in separate stages. This work aims to provide a wider perspective on the need to consider both mechanical and electromagnetic demands together in the design process. The paper provides an investigation of a Radial Flux PMSM (Permanent Magnet Synchronous machine) motor, targeted for a BLI propeller. The SPM (Surface Permanent Mounted) configuration has been chosen for its high power density and efficiency features. The focus is almost exclusively on the rotor component of the motor, with a preliminary introduction of geometry optimization results as a starting point for the design analysis.

\section{TOPOLOGY SELECTION AND GEOMETRICAL OPTIMIZATION}

The SPM has been chosen in the form of a Rim Driven topology to capture the boundary layers air-streams, Fig. 1. The design is geometrically constrained: forcing the active magnetic part inner hub diameter to $300 \mathrm{~mm}$. For the sake of clarity the rotating machine topology falls into the standard interior rotor category, having the fan embedded in the inner rotor structure, with standard outer slotted stator.

\begin{tabular}{ll}
\hline Rotor ID & $300 \mathrm{~mm}$ \\
Rated speed & $14000 \mathrm{rpm}$ \\
Power & $250 \mathrm{~kW}$ \\
$2 \mathrm{p}$ & 12 \\
$Q_{s}$ & 72 \\
Halbach-seg/pole & 4 \\
Targeted Current density & $12 \mathrm{~A} / \mathrm{mm}^{2}$ (rms) \\
DC-Link Voltage & $600 \mathrm{~V}$ \\
Operation & Constant speed at cruise \\
\hline
\end{tabular}

TABLE I: Main motor specifications

Rim drives are common in marine thrusters. Related benefits are the absence of mechanical couplings and a fan flow free from obstruction [6]. In this work the rim-driven rotor topology consists of a high strength solid steel shroud, on top of which PMs are positioned. To retain the PM segments, a Zylon filament sleeve wraps the entire rotor assembly. The motor specifications are given in Table I. A geometrical optimization of the electric motor 2D cross section is carried out, by means of a Genetic Algorithm. The fitness function minimizes electric loading and weight. Every optimization run explores a design space consisting of 40 generations with 100 


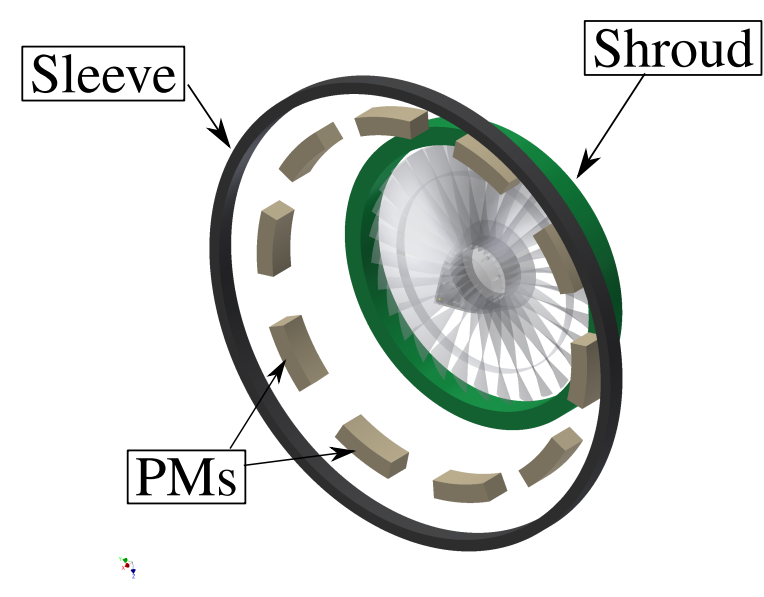

Fig. 1: Rim-Driven Machine Rotor Assembly

individuals per population. Choosing the electric loading with a fixed current density is adequate, as losses are dominated by winding copper loss. Assumptions, on top of the specifications given, are essential to comprehend the optimization conducted:

- The slots/poles combination has been dictated by previous investigation: Tab I

- One slot per pole short pitching: reduces space-harmonic

- Halbach-PM-Array chosen to maximize magnetic loading, reduce rotor core back flux size, reduction of losses, i.e. more sinusoidal variation of flux density within the air-gap

- Shaft-hub assembly consists of high strength solid magnetic steel

- The prototype will not have a fan design, rather a spoked geometry, pseudo-emulating a rim-driven shaped rotor

Although not prominently relevant for the scope of this paper the optimization assumed JNEX900 lamination steel from JFe Steel, and Rectangular Litz Wire type 8 for the stator winding, located within parallel open slots.

Pareto's optimization fronts of the HSHP-PMSM for (a) $\mathrm{NdFeB}$ and (b) SmCo PM arrays are compared in Fig. 2. $\mathrm{NdFeB}$ provides a higher power density because of its lower density, allowing thinner sleeves, and its higher energy product. Being fully encapsulated by retaining sleeve and end-plates at the rotor axial ends, NdFeB PM blocks are not affected by corrosion usually encountered in aerospace applications. Nevertheless, the choice has been made to use SmCo. Rotor losses are expected to be high and SmCo can handle much higher temperatures than $\mathrm{NdFeB}$ without losing magnetization properties.

Ultimately, the design-candidate from the Pareto's fronts is chosen assuming a maximum electric loading of $100000 \mathrm{~A} / \mathrm{m}$. This is deemed to be a suitable equivalent thermal level for the motor. Hence, a dominant solution in this respect is picked as soon as the Pareto front crosses this threshold, highlighted by the horizontal dashed line in both Fig. 2 (a) and (b). The aforementioned electric loading refers to a current peak value rather than the usual RMS value, being defined as per eq.

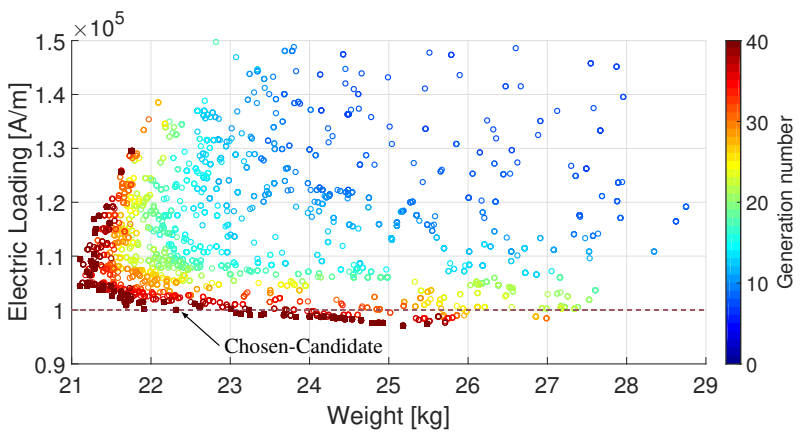

(a) NdFeB $42 U H$ Optimization

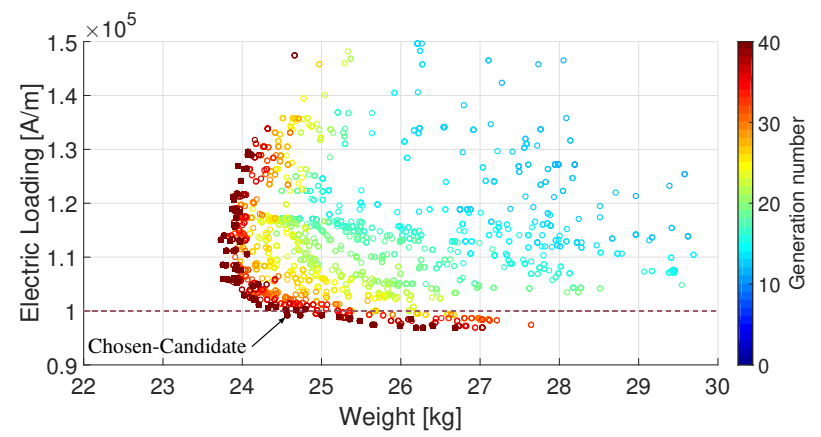

(b) SmCo Recoma 30 Optimization

Fig. 2: NdFeB (a) against SmCo (b) Electric loading vs Motor weight (active parts) - Pareto's fronts.

(1), where $k_{w}$ stands for the winding coefficient, $N_{s}$ for the number of conductors per series per phase, $\hat{I}_{s}$ the peak current, $D$ the stator inner bore diameter.

$$
\hat{K}_{s}=\frac{3 k_{w} N_{s} \hat{I}_{s}}{\pi D}
$$

\section{Mechanical Modelling}

Unlike marine applications, in which the rim-driven configuration runs at relatively low speed, the high tip velocities for this aerospace application play a predominant role in the rotor design. It must be structurally robust to prevent any failure.

- Maximum tensile stress in every rotor component must not exceed the material yield strength;

- PMs should sustain the sleeve compression at zero speed

- Lift-off condition must not occur for the PMs (at the shaft/hub OD)

- Rotor-dynamically induced vibration modes should occur above the machine maximum speed

\section{A. Sleeve Design and Verification}

The sleeve size dimensioning is performed according to an extended analytical model embedded in the optimization process. Rotor assembly material properties are given in Table III. The rotor has been simulated by means of 3D mechanical FEA, to check component stresses both at zero and over-speed $(+20 \%$ than nominal) conditions, as per Fig. 3. The figure shows the simulated domain, restricted to a single axially-half cut spoke, exploiting the geometrical symmetry. At zero-speed 
the radial stress concentration occurs at the spoke smallest cross section, while the tensile stress peak value belongs to the inner diameter of the retaining sleeve. For the overspeed condition, the arrows points to the real peak value of stresses (numerical errors affected sharp corners that for the sake of simulation time were left in the modelled geometry, but are not present in reality). The tensile stress is again maximum around the inner bore of the sleeve. Radial stress concentration affects the smallest spoked cross section, but now in expansion (positive pressure). The outer shaft diameter did not experience positive pressure values, witnessing no lift-off. Careful consideration must be made addressing the actually unrealistic retaining sleeve modelling: The Zylon is an anisotropic composite fibre, but it has been modelled as a solid medium. This is due to difficulties in accurate modelling of composite fibres in FEA codes, as well as unavailability of anisotropic behaviour data for this specific fibre. Results must then be taken cautiously in terms of reliability. Nonetheless, no particular stress concentration was observed in the other components, apart from the fibre, encouraging the assumption of a safe operation within the machine operating speed range.

\section{B. Bearings}

A key aspect for the high-speed compliance of the electrical machine is the adoption of Super-Precision-Bearings. Bearings play a significant role in rotor-dynamic interaction, as they effectively behave as springs sustaining the rotor [7]. A bearing choice is not only dictated by speed, but rather a combination of speed, lubricant type, loads and bearing life. The choice undertaken in this study considers grease lubricant. In a real BLI Rim-Driven motor, most of the load experienced by the bearings will be given by the axial air flow. For this prototype such a situation has been discarded and a choice considering better radial load withstanding has been preferred for the Drive-End. Therefore, a pair of angular contact bearing backto-back is employed at the drive end, whereas a single axially loaded angular contact bearing is considered for the NonDrive-End. A cross section of the inner shaft with bearing arrangement is given in Fig. 4.

\section{Rotor-Dynamic Stability}

Any rotating system is prone to exhibit instability, mostly due to resonance modes occurring around specific frequencies at which the rotor component is self-excited. This rotor geometry exhibits a very low torsional stiffness. This is due to the unusually low aspect ratio (rotor-length over rotor outer diameter), and is further exacerbated by the presence of spokes. As a proof of concept, spokes are not essential, leaving a theoretical shape of a full disk. However, they are necessary for the manufacturing process, in order to allow clamping tools to be put in place when the retaining bandage is wrapped around the rotor. The torsional vibration seems to occur outside of the operating speed range (0-14000rpm). No coupling assumptions have been considered in the modelling, between the different rotor assembly components. This is equivalent to assume a worse case scenario as the stiffness is

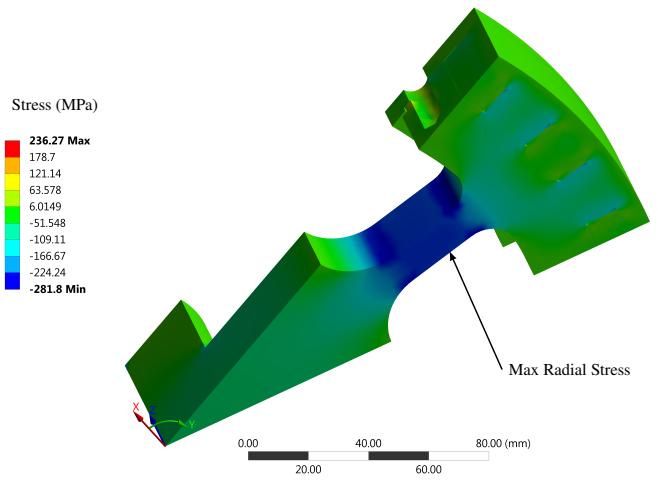

(a) Zero-speed radial stress

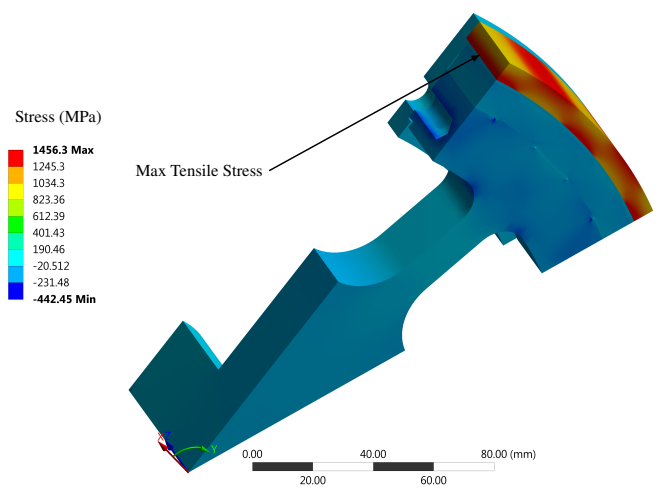

(b) Zero-speed tensile stress

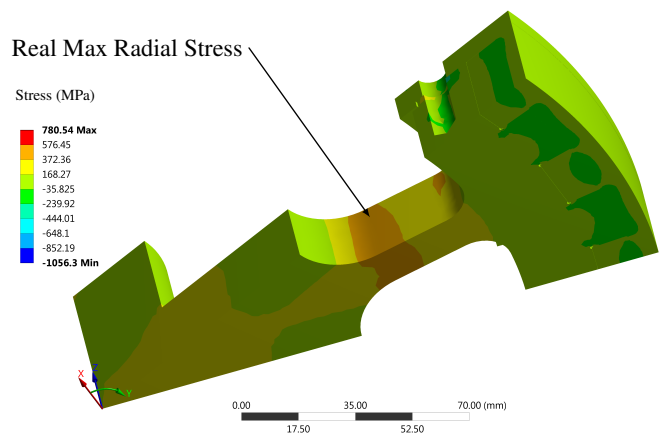

(c) Over-speed radial stress

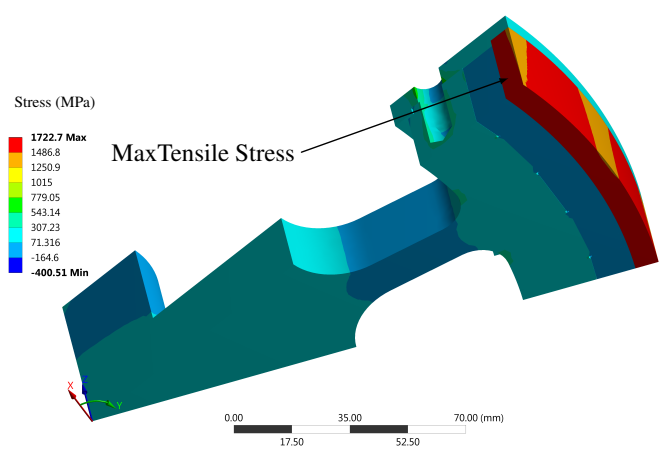

(d) Over-speed tensile stress

Fig. 3: Structural analysis

reduced with respect to the reality: A bigger stiffness for the same moment of inertia delivers lower resonance frequencies. 


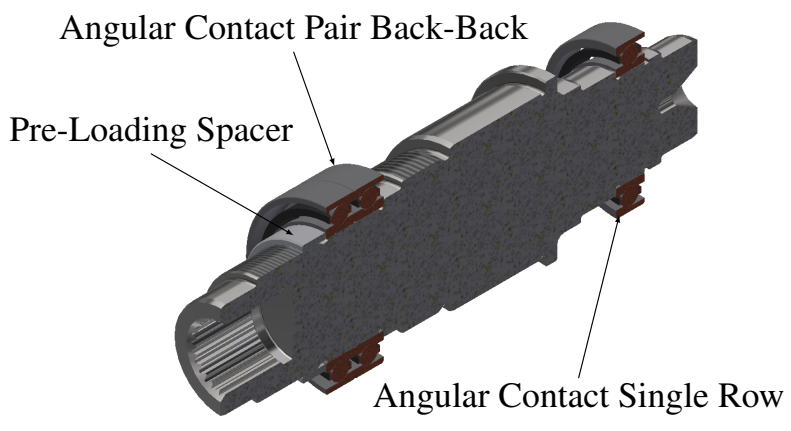

Fig. 4: Half of rotor shaft.

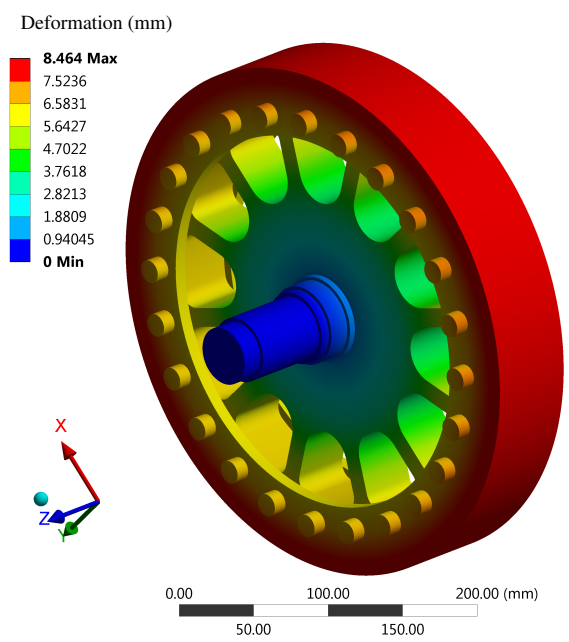

(a) Torsional vibration mode

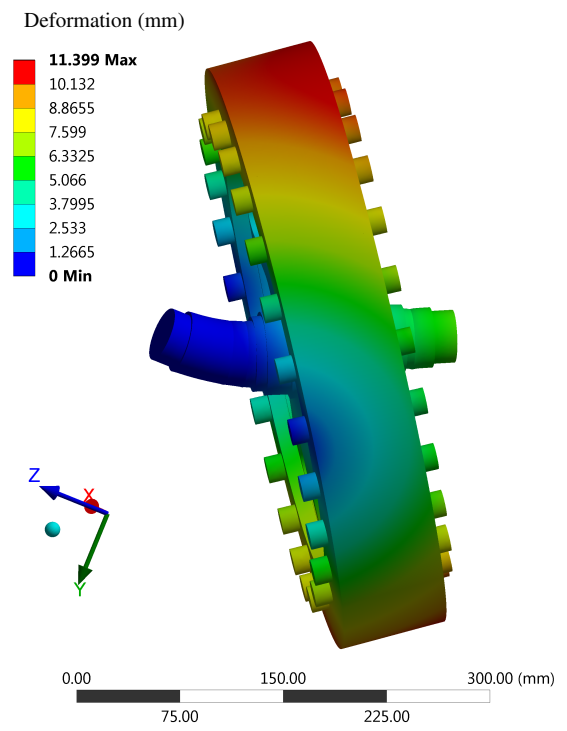

(b) First bending mode (13X)

Fig. 5: Torsional and first bending modes.

Fig. 5 and Fig. 6 show the first 4 resonance mode shapes. The bearing supports have been modelled as localized springs with isotropic stiffness along the radial cross section axis. Stiffness values were provided by the manufacturer based on overall

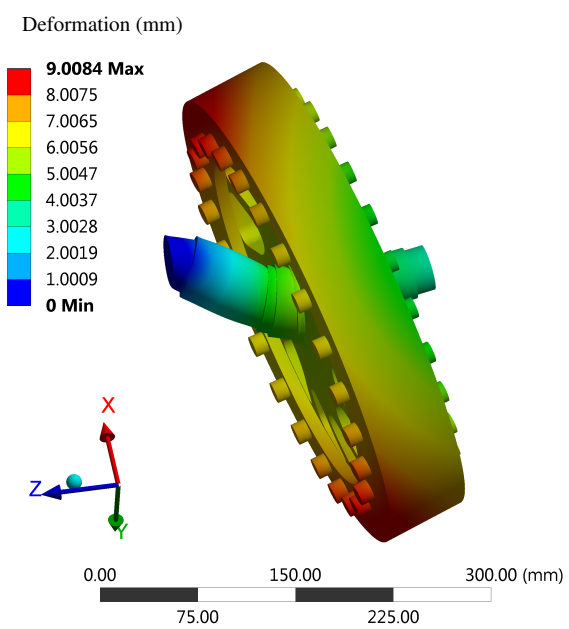

(a) Second bending mode (9X)

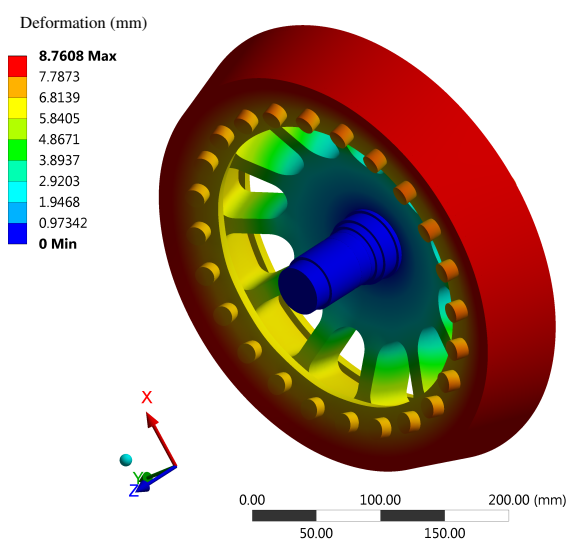

(b) Membrane shaped vibration mode (6X)

Fig. 6: Second bending and membrane shaped modes.

rotor mass and bearing location with respect to the centre of mass. As specified in Tab. II the torsional vibration occurs at low speeds (equivalent to $3800 \mathrm{rpm}$ ), whereas higher modes are positioned far off in the frequency spectrum.

\begin{tabular}{llll}
\hline Mode & Vibration type & Frequency $[\mathrm{Hz}]$ & speed [rpm] \\
\hline 1 & Torsional & 338 & 20280 \\
2 & Bending & 409 & 24540 \\
3 & Second Bending & 781 & 46860 \\
4 & Membrane-shaped & 1150 & 69000 \\
\hline
\end{tabular}

TABLE II: Natural frequencies

\section{Details on Manufacturing}

The adoption of a unique shaft-hub assembly has been discarded for impracticability and too high machining cost. The choice adopted employs a shrunk fit shaft within a spoked hub. Such an assembly should not affect the overall rotordynamic behaviour, at least as long as slipping does not occur between shaft and hub. Appropriate interference has been chosen to compromise between material yield strength at the 


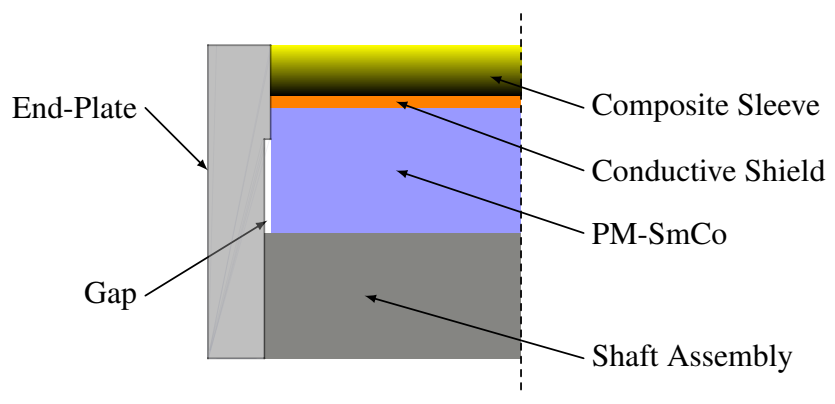

Fig. 7: Half of rotor cross section.

coupling bore, and expansion due to centrifugal forces at rated speed.

Referring to the rotor components arrangement represented in Fig. 7, a crucial aspect is having a contact point between end plate and PM at the PM-OD only. In this way the end-plates are not flexed outward by a lever point at or below the bottom of the PM. The lever point arises because the plates will be bolted at the outer shaft-assembly axial ends. With a clamping force due to the bolts at the lower edge of the end-plate a reactive force works to close the gap at PM-OD. Without a gap the reactive force is still located at the lower PM edge, so that there is a lever effect trying to create a gap at the PM OD.

The choice of end plate material is essential as a trade off between strength and electrical conductivity. Available choices considered were Non-magnetic-Stainless steel 316, Titanium Grade 5 (Ti6Al4V) and Inconel 718. The last was ruled out for enormous effort required on the manufacturing side, and the very high cost. The first option is not feasible in terms of strength capabilities, although providing the lowest conductivity possible. On the other hand Titanium offers the best compromise, due to a relatively limited electrical conductivity, along with a very high tensile strength and low density.

The Zylon fibre constitutes a strength improvement with respect to a more standard carbon fibre composite. Nonetheless, due to still unknown thermal, light and moisture sensitivity properties the sleeve will be wrapped with a protective layer of carbon fibre on top and bottom of the rotor assembly.

\section{Electromagnetic Modelling}

Slot, poles and short pitching combination constitute the most suitable choice in terms of spatial harmonics content. The Halbach array provides a high fundamental torque component, due to the highly sinusoidal flux linkage/back-emf, [8], [9]. Fig. 8 (a) presents the approximate 3D finite element geometry used to estimate the overall inductance, which includes 3D effects as the machine is very short axially.

Fig. (9) proves the suitability of the Halbach array showing the 3 dimensional No-Load flux linkage across the three phases of the machine (a), together with the harmonics magnitude (b) in logarithmic scale. It is clearly noticeable as the first harmonic completely dominates the waveform, being essentially sinusoidal.

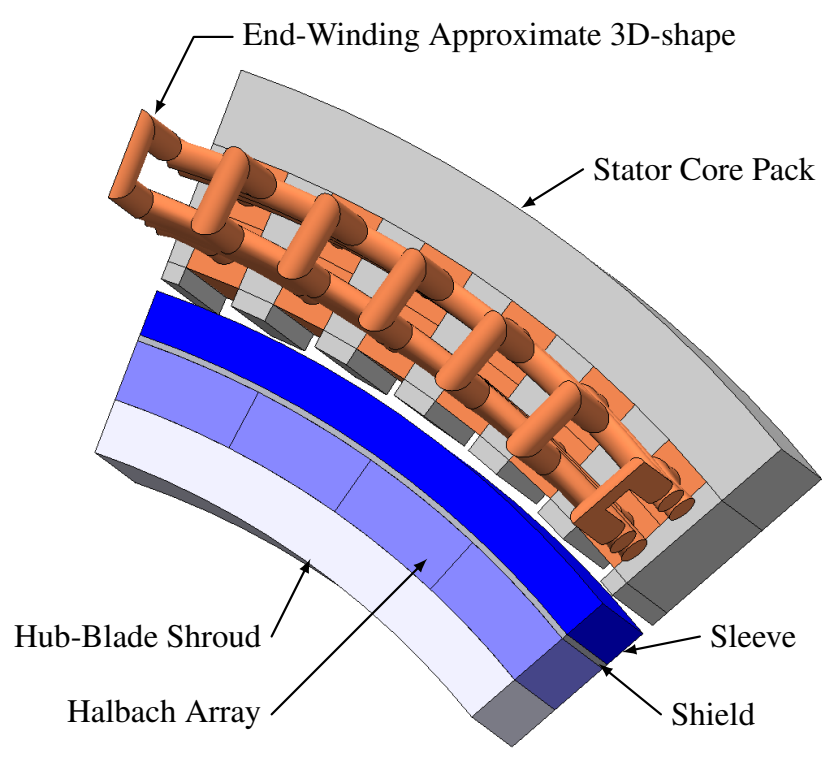

Fig. 8: 3D geometry used for FEA analysis

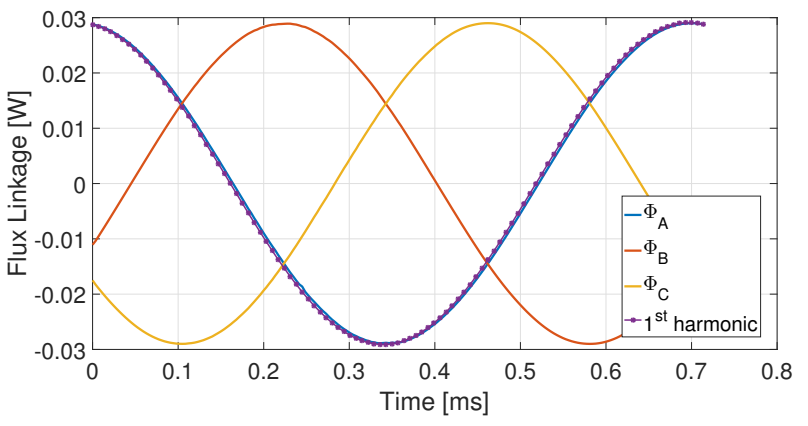

(a) No Load flux linkage over time

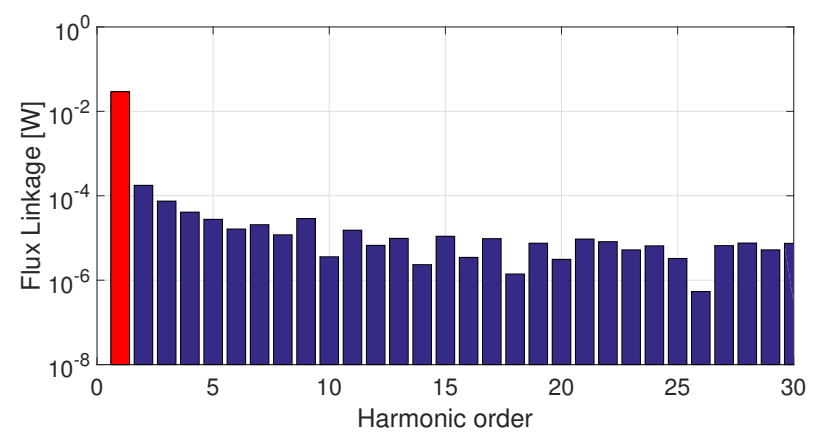

(b) Log scale of flux linkage harmonic order

Fig. 9: No load flux linkage from Halbach array.

Nonetheless, rotor losses are high when ripple is included in the armature current spectrum. Even though employing a higher number of axial segments [10], decreases the overall losses because of an increase in the eddy current path, it conversely increases the amount of rotor loss caused by time harmonics whose pole pitch is wider than space harmonics, resulting in a higher depth of penetration into the rotor. Eddy currents tend to circulate in a skin at the PM edges rather than on the outer radial surface as occurs with space harmonics. 


\begin{tabular}{lllll}
\hline Material & Material & Yield Stress & Young Modulus & Therm. Expansion Coeff. \\
\hline Shaft/hub & Stainless Steel 17-4PH & $900 \mathrm{MPa}$ & $200 \mathrm{GPa}$ & $12 \mathrm{e}-61 / \mathrm{K}$ \\
\hline Magnets & SmCo-Recoma 30 & $140 \mathrm{MPa}$ & $120 \mathrm{GPa}$ & $8 \mathrm{e}-61 / \mathrm{K}$ \\
\hline Sleeve & Zylon/Carbon Fibre & $>3000 \mathrm{MPa}$ & $186 \mathrm{GPa}$ & $-1 \mathrm{e}-61 / \mathrm{K}$ \\
\hline End Plates & Titanium - Ti6Al4V & $1100 \mathrm{MPa}$ & $114 \mathrm{GPa}$ & $9 \mathrm{e}-61 / \mathrm{K}$ \\
\hline
\end{tabular}

TABLE III: Rotor Parts Mechanical Properties

This is observable in Fig. 10, where an axial cross section of a PM is post-processed from a 2D FEA. No PM rotor can withstand such losses, without causing permanent loss of magnetization properties due to the increased temperature. To tackle this issue either an extremely fine segmentation (employing segments thinner than the skin depth related to the time-harmonic frequency) or PM screening against harmful asynchronous harmonics could be utilized. The former solution is too challenging, both in terms of manufacturing and cost, consequently the latter approach has been chosen.

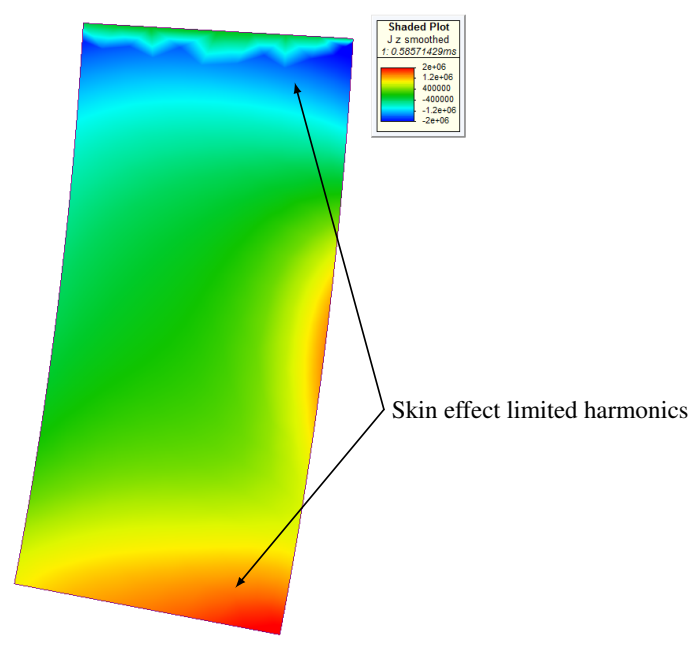

Fig. 10: Single PM segment showing ECs distribution due to time harmonics

\section{A. Inclusion of Drive THD}

Given the high fundamental operating frequency, a low stator inductance is expected. Considering a Voltage Source Inverter fed motor, the current ripple is anticipated to have a significant impact on the motor performance [11]. In order to do so, resistance, inductance and PM flux values must be estimated, Tab. IV. The resistance has been computed analytically without considering increments due to $\mathrm{AC}$ effects: The wire resistance employed here has not got as a significant impact as the inductance. The PM flux is assumed for $100{ }^{\circ} \mathrm{C}$ operation. The air-gap exhibits a constant reluctance along the $\mathrm{d}$ and $\mathrm{q}$ axis due to the SPM Halbach array.

The derived waveform with intrinsic time-harmonics ripple and corresponding harmonic content is provided for both standard $d q$ SPM equivalent circuit model in Fig. 11. Switching frequency has been assumed 10 and 20 times the fundamental

\begin{tabular}{cc}
\hline$R_{s}$ & $6.3 \mathrm{~m} \Omega$ \\
$L_{d}=L_{q}$ & $32.5 \mu \mathrm{H}$ \\
$\psi_{\mathrm{PM}}$ & $0.29 \mathrm{~Wb}$ \\
\hline
\end{tabular}

TABLE IV: Electro-magnetic parameters

for comparison (14 and $28 \mathrm{kHz}$ ). It is clearly visible a non sinusoidal high harmonic content.

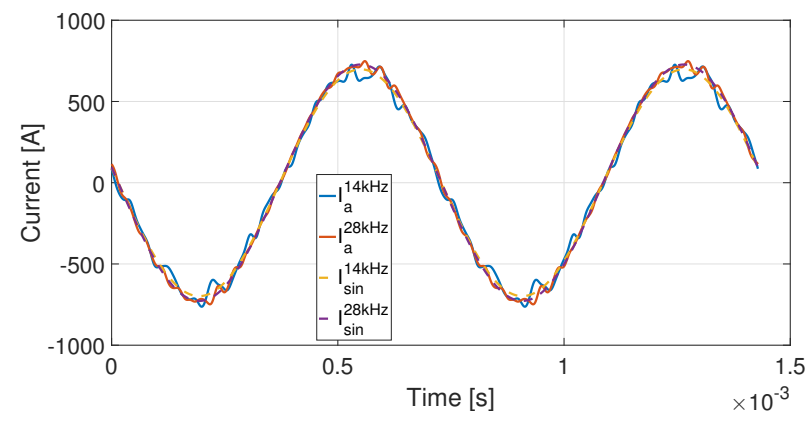

(a) Ripple at $14 \mathrm{kHz}$ switching

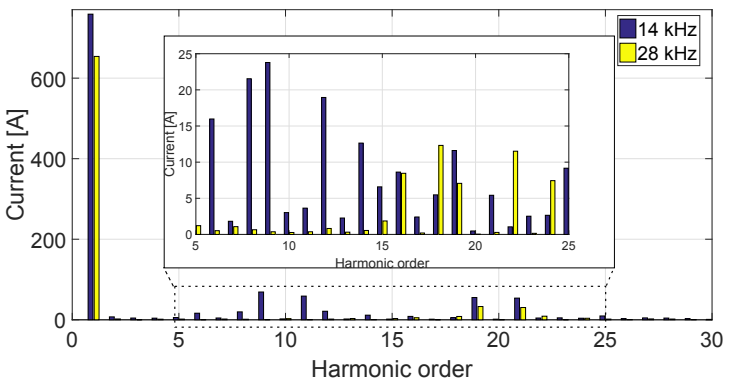

(b) Ripple at 14 and $28 \mathrm{kHz}$ switching

Fig. 11: Standard $d q$ circuit dynamic model current waveform.

\section{B. Shielding and eddy currents evaluation}

A sizing for the shield has been attempted, comparing copper and aluminium as screening material. The sizing has been performed assuming a harmonic distortion computed by means of the standard $d q$ dynamic model for the PM machine. The machine is simulated in motor operation at constant rated speed, i.e. no outer speed loop is employed. The current control is the implemented strategy. A 2-DOF PI controller, based on the robust pole placement technique proposed in [12] is employed The VSI switching scheme is based on Continuous Space Vector Modulation with equal sampling 
and switching frequency. The switching frequency assumed is 10 and 20 times the fundamental in MTPA condition: $\pi / 2$ current angle, full $q$ axis current. The trend of loss for different shield thickness is shown in Fig. 12. Copper seems to give a slight advantage on overall loss reduction. Moreover, after $1 \mathrm{~mm}$ thickness the loss decrease is not relevant enough, in order to justify a larger thickness to be employed: This will be detrimental for the torque density and sleeve stresses.

Eddy current losses are intrinsically a 3 dimensional effect. Employing Titanium rings effectively adds up a conductive component subject to asynchronous flux density harmonics. This will generate additional losses. An estimation of these has been attempted by means of FEA. Losses have been computed for $14 \mathrm{kHz}$ switching frequency with single inductance (over frequency) dynamic model (standard dynamics for SPM motor) control scheme. The shield is effectively screening the PMs and the conductive shaft. Considerable losses are detected in the end plate. Means to reduce these high losses on the endplates are needed. Due to space restrictions, and modifications limitations, a fillet feature has been investigated along the rotor outer periphery. This releases the end-plate of a certain amount of loss, Fig. 13 (b). For machinability purposes an even easier choice of material removal will be actually employed as shown in Fig. 13 (a). It is imperative for the drive to operate at high switching frequencies unless a likely unsustainable loss is generate in the rotor.

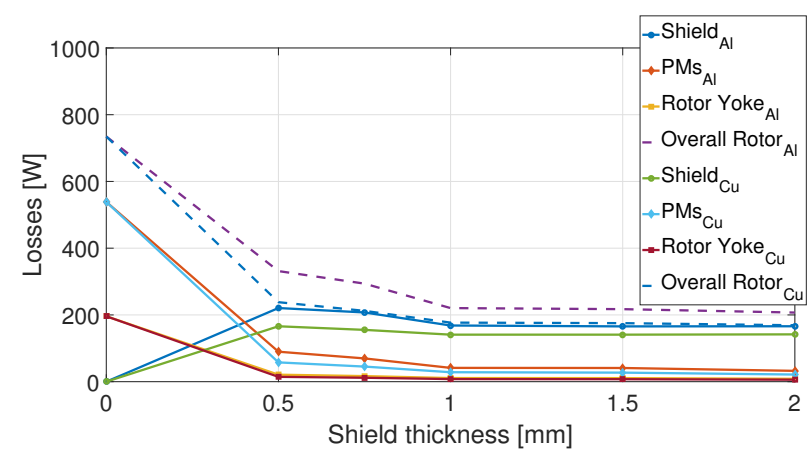

Fig. 12: $\mathrm{Al}$ vs $\mathrm{Cu}$ shielded rotor losses. 2D-FEA analysis

\section{Shielding utilization implications}

When a shielding component is employed within an electromagnetic assembly it affects the device, as parasitic eddy currents come into play in the overall power balance. A SPM motor equipped with a shield will experience a behaviour similar to the wound field synchronous generator with damper cages [13]. A progressively increasing frequency will reduce the flux linkage between the stator and rotor, decreasing an already low inductance value. Inductance trends can be described as in [3] and [14] by complex Eq. 2. A frequency response analysis is deemed to be useful for evaluating this effect, emulating the Standstill frequency-response test. A 3D Time-Harmonic analysis has been carried out in order to obtain the inductance variation over the frequency spectrum. Since

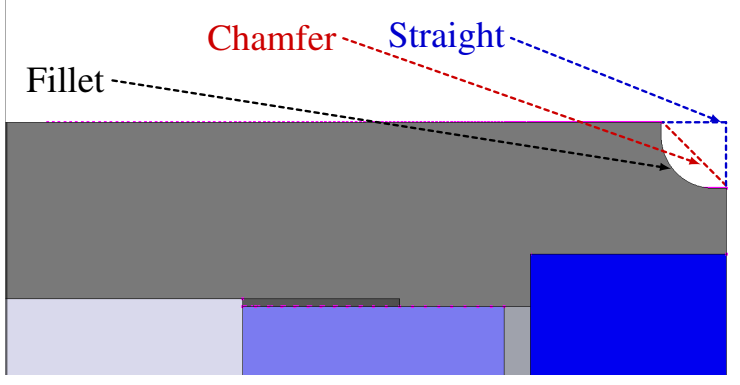

(a) End plate edge

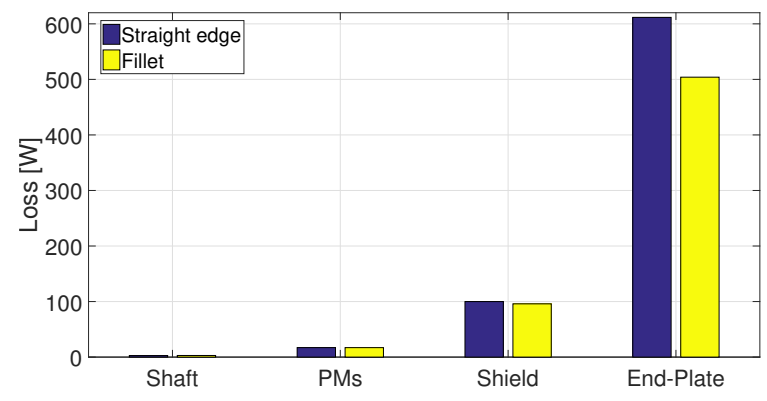

(b) Rotor Losses split

Fig. 13: End plate OD edge and related loss 3D estimation.

the magnetic loading is relatively low, a series of linear TimeHarmonic models have been used to estimate the phenomena.

$$
L_{d}(\mathrm{j} \omega)=L_{d}(0)\left[\frac{1+\mathrm{j} \omega T_{d}^{\prime \prime}}{1+\mathrm{j} \omega T_{d 0}^{\prime \prime}}\right]
$$

The Levenberg Marquardt algorithm has been employed to estimate the parameters from an integer number of 3D TimeHarmonic FE analysis. Real and imaginary components are shown with their correspondent fitting, for $\mathrm{Al}, \mathrm{Cu}$ and without any shield in Fig. 14. Employing the first-order complex system described by eq. (2) provides a rough fitting shown in Fig. 14.

Due to manufacturing choices, explained in the proceedings, aluminium was deemed to be the most suitable material for shielding. In light of the recognized inductance drop with the frequency, clearly detectable in Fig. 14, it is imperative that, when assuming a very high switching frequency, to limit the current ripple and the consequent rotor losses as much as possible.

\section{Details on manufacturing}

The higher number of Halbach array segments, the more sinusoidal magnetic field shape is obtained in the air-gap. Four magnetized segments per pole has been chosen as a compromise between a reasonably high flux density $\hat{B}_{g}$ and manufacturing cost.

Aluminium was chosen for the better machinability, reduced cost and weight, whilst still maintaining a good thermal and electrical conductivity. As shown in Fig. 14 a copper shield would produce a larger inductance drop with the increasing frequency, generating more current ripple. 


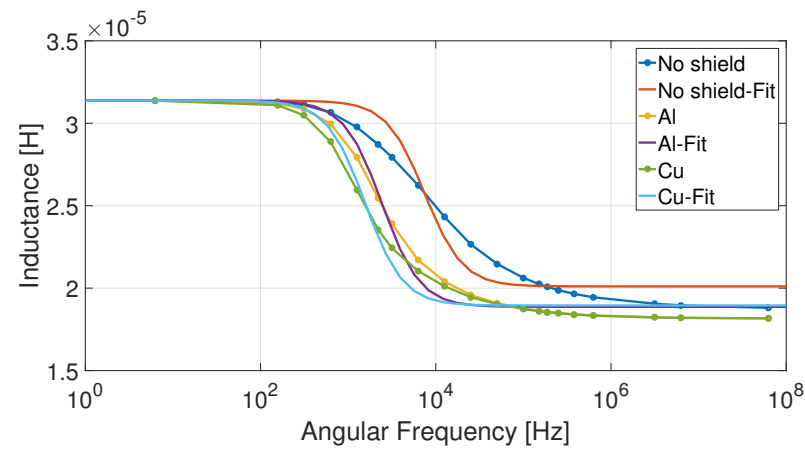

(a) $\operatorname{Re}\left(L_{d}(\omega)\right)$

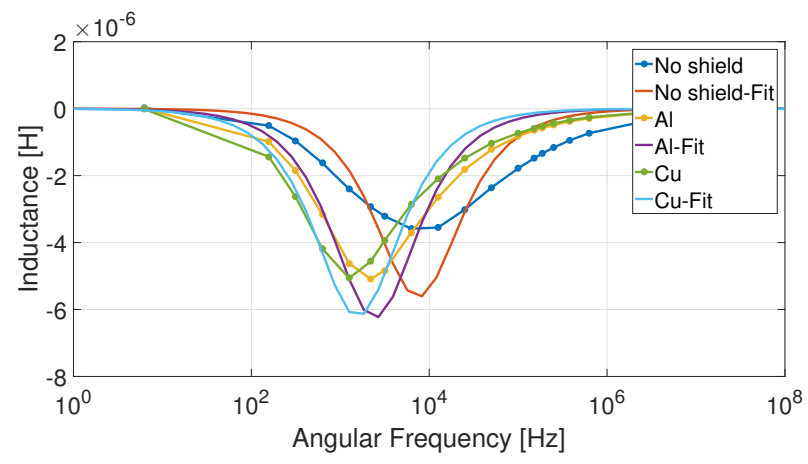

(b) $\operatorname{Im}\left(L_{d}(\omega)\right)$

Fig. 14: Inductance components with First Order fitting

\section{CONCLUSiOnS}

In this research, emphasis has been put on how HSHP electrical machines could embody remarkable design challenges. Stresses and rotor-dynamics, alongside with electromagnetic rotor losses estimation have been addressed. Design challenges have been tackled from different physics simultaneously. It is clear as such a design cannot be driven at low switching frequencies due to an intrinsically low inductance that is responsible for high current ripple in VSI drives, in turn resulting in very high losses. This has been proven by extracting lumped electromagnetic parameters of the machine and emulating a Voltage source driven current controlled equivalent 1D model motor.

Mechanical stresses on active and non active components must be checked to ensure a safe operation. Rotor-dynamics instability must be ruled out of the operational range. Shielding is proposed as an extra component in order to protect the PM from excessive losses and over-heating. Sizing of the latter and design solutions accommodating machinability and suitable assembly have been provided.

The implications of screens, and in general conductive components, within the rotating part has risen the issue of a more accurate inductance modelling. A more mathematically sound dynamic model of a PMSM with conductive rotor part is currently part of further research work, extending what briefly presented in [3]. This aims to extend Eq. 2 fitting to a second order system and augment the equivalent circuit accordingly. In turn, it will represent a new mean for a more accurate electromagnetic analysis of frequency varying inductance machines, or synchronous motors equipped on purpose with conducting parts, such as the on-line starting motors.

Finally, future research developments will comprise prototype building and testing of the presented motor design, to validate the most of the electro-mechanics aspects treated. A complete thermal model for the motor, linked strictly with the losses level, is at the moment missing, and will be attempted despite the inevitable approximations required.

\section{REFERENCES}

[1] M. van der Geest, H. Polinder, J. A. Ferreira, and M. Christmann, "Power density limits and design trends of high-speed permanent magnet synchronous machines," IEEE Transactions on Transportation Electrification, vol. 1, no. 3, pp. 266-276, Oct 2015.

[2] H. Polinder and M. J. Hoeijmakers, "Effect of a shielding cylinder an the rotor losses in a rectifier-loaded pm machine," in Conference Record of the 2000 IEEE Industry Applications Conference. Thirty-Fifth IAS Annual Meeting and World Conference on Industrial Applications of Electrical Energy (Cat. No.00CH37129), vol. 1, Oct 2000, pp. 163-170 vol.1.

[3] D. J. B. Smith, S. M. Lambert, B. C. Mecrow, and G. J. Atkinson, "Interaction between pm rotor design and voltage fed inverter output," in 6th IET International Conference on Power Electronics, Machines and Drives (PEMD 2012), March 2012, pp. 1-6.

[4] M. M. J. Al-Ani, G. Vakil, and C. Gerada, "Electrical machine rotor shielding for low cost electrical drive," in 2018 IEEE Transportation Electrification Conference and Expo (ITEC), June 2018, pp. 38-43.

[5] M. M. J. Al-ani, S. M. Barrans, and J. Carter, "Electromagnetic and mechanical analysis of high speed spm rotor with copper shield," in 2017 IEEE International Electric Machines and Drives Conference (IEMDC), May 2017, pp. 1-8.

[6] P. M. Tuohy, A. C. Smith, M. Husband, and P. Hopewell, "Rim-drive marine thruster using a multiple-can induction motor," IET Electric Power Applications, vol. 7, no. 7, pp. 557-565, Aug 2013.

[7] N. Uzhegov, E. Kurvinen, J. Nerg, J. Pyrhnen, J. T. Sopanen, and S. Shirinskii, "Multidisciplinary design process of a 6-slot 2-pole highspeed permanent-magnet synchronous machine," IEEE Transactions on Industrial Electronics, vol. 63, no. 2, pp. 784-795, Feb 2016.

[8] M. Galea, L. Papini, H. Zhang, C. Gerada, and T. Hamiti, "Demagnetization analysis for halbach array configurations in electrical machines," IEEE Transactions on Magnetics, vol. 51, no. 9, pp. 1-9, Sep. 2015.

[9] P. H. Mellor and R. Wrobel, "Optimization of a multipolar permanentmagnet rotor comprising two arc segments per pole," IEEE Transactions on Industry Applications, vol. 43, no. 4, pp. 942-951, July 2007.

[10] J. D. Ede, K. Atallah, G. W. Jewell, J. B. Wang, and D. Howe, "Effect of axial segmentation of permanent magnets on rotor loss in modular permanent-magnet brushless machines," IEEE Transactions on Industry Applications, vol. 43, no. 5, pp. 1207-1213, Sept 2007.

[11] E. P. Wiechmann, P. Aqueveque, R. Burgos, and J. Rodriguez, "On the efficiency of voltage source and current source inverters for high-power drives," IEEE Transactions on Industrial Electronics, vol. 55, no. 4, pp. 1771-1782, April 2008

[12] M. Hinkkanen, H. Asad Ali Awan, Z. Qu, T. Tuovinen, and F. Briz, "Current control for synchronous motor drives: Direct discrete-time pole-placement design," IEEE Transactions on Industry Applications, vol. 52, no. 2, pp. 1530-1541, March 2016.

[13] K. W. Klontz, T. J. E. Miller, M. I. McGilp, H. Karmaker, and P. Zhong, "Short-circuit analysis of permanent-magnet generators," IEEE Transactions on Industry Applications, vol. 47, no. 4, pp. 1670-1680, July 2011.

[14] T. M. J.R. Hendershot, Design of Brushless Permanent-Magnet Machines, 2010. 